Itinéraires Itinéraires

Littérature, textes, cultures

2019-2 et $3 \mid 2019$

Corps masculins et nation : textes, images, représentations

\title{
Corps noirs, virilité et pouvoir dans la littérature médicale à l'époque coloniale
}

Black Bodies, Virility and Power in Medical Literature in a Colonial Context

\section{Delphine Peiretti-Courtis}

\section{(2) OpenEdition}

\section{Journals}

Édition électronique

URL : http://journals.openedition.org/itineraires/6605

DOI : 10.4000/itineraires.6605

ISSN : 2427-920X

Éditeur

Pléiade

Référence électronique

Delphine Peiretti-Courtis, « Corps noirs, virilité et pouvoir dans la littérature médicale à l'époque coloniale », Itinéraires [En ligne], 2019-2 et 3 | 2019, mis en ligne le 26 novembre 2019, consulté le 15 décembre 2019. URL : http://journals.openedition.org/itineraires/6605 ; DOI : 10.4000/itineraires.6605

Ce document a été généré automatiquement le 15 décembre 2019.

\section{cc) $(1) \ominus$}

Itinéraires est mis à disposition selon les termes de la licence Creative Commons Attribution - Pas d'Utilisation Commerciale - Pas de Modification 4.0 International. 


\title{
Corps noirs, virilité et pouvoir dans la littérature médicale à l'époque coloniale
}

\author{
Black Bodies, Virility and Power in Medical Literature in a Colonial Context
}

Delphine Peiretti-Courtis

1 Après la Révolution française, l'idée de nation se structure et se définit à partir de plusieurs critères : le sentiment d'appartenance à une même communauté, un territoire commun, une identité et des valeurs partagées, mais également à partir de la notion de souveraineté nationale. Cette souveraineté est détenue par ceux qui peuvent voter : les hommes. La nation s'illustre donc et affiche sa puissance grâce à ses hommes et à leur virilité. L'homme blanc, de la haute société française, est celui qui incarne la norme en termes de virilité et de pouvoir. Face à l'extérieur, la nation française doit se présenter comme forte, puissante, et par conséquent être portée par des hommes dotés d'une masculinité accomplie. À l'intérieur du territoire, la mise en lumière des éléments déviants et jugés inférieurs - hommes des classes populaires, ouvriers affaiblis par l'alcool et le vice, mais aussi paysans considérés comme rustres et ignorants de la politique - contribue, par un jeu de miroir, à renforcer le prestige et le pouvoir des hommes en charge de la responsabilité du pays et de la nation. Les ouvrages de Régis Revenin sur l'histoire des masculinités à partir de 1789 (Revenin 2007) et l'œuvre majeure coordonnée par Alain Corbin, Jean-Jacques Courtine et Georges Vigarello sur l'histoire de la virilité (Corbin, Courtine et Vigarello 2011) éclairent ces questions. Le citoyen aisé, électeur et éligible, doit constituer un exemple pour l'ensemble des Français. Il incarne la civilité, la modération, la pugnacité ainsi que les valeurs du travail, de la famille et de l'épargne comme le montre notamment Anne-Marie Sohn dans son ouvrage Sois un homme, la construction de la masculinité au XIXe siècle (Sohn 2009). Cet homme symbolise la civilisation arrivée à son paroxysme. De la même manière que ce modèle de virilité doit s'étendre en France à toutes les classes, il doit, par le biais de l'expansion coloniale, se diffuser au sein des colonies, à toutes les races. Cette étude s'inscrit dans les recherches sur l'intersectionnalité, un concept popularisé en France 
par Colette Guillaumin (1992) puis Elsa Dorlin (2006) et qui permet d'analyser l'histoire à travers le prisme des interactions entre la race, le sexe et la classe. L'histoire des masculinités en contexte colonial s'est développée au sein de l'historiographie du genre et plus particulièrement de celle la virilité (Sinha 1995, Connell 2005, Taraud 2011). Par exemple, selon Christelle Taraud dans l'Histoire de la virilité parue en 2011 (Taraud 2011), l'effémination des hommes d'Afrique du Nord a permis de justifier la prise de pouvoir des Français sur ces territoires. Mrinalini Sinha évoquait déjà dans une étude historique pionnière en 1995 la façon dont la virilisation du colonisateur britannique était corrélée à la féminisation de l'Indien dans un jeu de miroir des rapports de pouvoir au moment de la colonisation (Sinha 1995). Ce thème a également été traité par Elsa Dorlin pour analyser les relations entre esclaves et maîtres durant la période esclavagiste (Dorlin 2006).

2 Cette analyse a pour but de démontrer que l'effémination de l'homme noir, au sein de la littérature médicale - dictionnaires de médecine, monographies médicales, traités d'hygiène, articles et rapports de médecins coloniaux - au cours de la période coloniale, a contribué à redorer la virilité de l'homme blanc et à légitimer la tutelle coloniale. Elle vise également à mettre en lumière la réapparition d'un autre stéréotype à la fin du XIX siècle, hérité de la période esclavagiste, celui d'un homme noir viril, fort et robuste. Cette représentation a notamment été diffusée à une période où il fallait démontrer que les Africains pouvaient servir la nation et l'Empire français.

\section{Effémination de l'homme noir et domination coloniale}

3 Afin de conforter la puissance et la supériorité des hommes de la nation française, l'homme des territoires étrangers colonisés a été défini dans la littérature médicale du $\mathrm{XIX}^{\mathrm{e}}$ siècle par sa faiblesse, son déficit d'autorité, voire son aspect féminin ou infantile pour les peuples africains (Virey 1817, Letourneau 1869, Montandon 1933). Ces sociétés, considérées comme étant peuplées d'hommes faibles et impuissants sont par ailleurs jugées comme étant des nations faibles et empreintes d'un désordre politique et social général. Le genre et les frontières entre les sexes sont décrits comme flous et troublés, la sexualité débridée et la moralité douteuse. L'homme viril de la nation française, celle des Lumières et de la Révolution française, celui qui s'est battu pour sa liberté et a imposé le principe de souveraineté nationale, revendique donc la mission de transmettre ce modèle aux autres peuples et notamment à ceux dont on considère que l'autorité masculine est défaillante. La nation française, et particulièrement dans sa dimension expansionniste, prend les traits d'un homme, colonisateur et civilisateur puissant et viril, prêt à apporter les Lumières à des populations supposées inférieures. Tandis que la Nation domine et protège, la République est féminisée et personnifiée sous les traits de Marianne, une figure maternelle et rassurante.

4 Dans les théories et hiérarchies raciales édictées par les médecins tout au long du XIXe siècle (Virey 1817, Broc 1836, Clavel 1860, Broca 1861, Topinard 1879), la race noire est présentée comme une race féminine à plusieurs titres. Premièrement, les hommes et les femmes noirs se distingueraient peu physiquement et intellectuellement. Le corps masculin est décrit comme glabre, le corps féminin musclé, la sexualité paraît irrépressible dans les deux sexes tandis que le crâne et le cerveau semblent similaires chez l'homme et la femme (Virey 1824, Broc 1836, Broca 1861, Montandon 1933). Les médecins en déduisent un développement intellectuel identique et, par conséquent - la 
femme étant perçue comme biologiquement inférieure à l'homme - une intelligence inférieure à celle de l'homme blanc (Cabanis 1802, Virey 1824, Topinard 1879, Le Bon 1898).

5 Les cerveaux seraient également conformés de manière différente selon les races et prédisposeraient les êtres à des facultés divergentes. Selon de nombreux savants tels le naturaliste Julien-Joseph Virey, ou le chirurgien et anthropologue français Paul Broca, la conformation du cerveau des individus africains s'apparenterait en tout point à celui des femmes dans toutes les races. Les aires cérébrales destinées à l'émotion, aux sens ou à la sexualité y seraient prééminentes à l'inverse du cerveau des hommes de race blanche dont les zones dévouées à la raison et à l'intellect seraient surdéveloppées. Si la thèse des localisations cérébrales est développée par Gall, médecin allemand à l'origine du développement de la phrénologie dans les années 1810, elle est ensuite appuyée et corroborée par Broca au milieu du XIX siècle. Ce sont des savants, médecins, naturalistes ou anthropologues, intéressés par l'étude de la diversité humaine, qui appliquent la thèse des aires cérébrales différenciées aux races dans la première moitié du XIX ${ }^{e}$ siècle. Ils contribuent donc, par leurs études, à accréditer l'idée que l'homme noir est un être efféminé et que ses penchants sexuels ou sa prédisposition aux instincts primaires sont la conséquence d'un déterminisme biologique et racial, par conséquent immuable. Ainsi, de nombreuses descriptions parues dans des dictionnaires médicaux et rédigées par des savants tels que Virey $(1817: 210,257)$, Broca, Letourneau (1869 : 524-534), ou encore Pittard (1927: 67) vont attester, pendant plus d'un siècle, des années 1810 aux années 1920, de l'aspect féminin du crâne des Africains. En 1933, le médecin et anthropologue Georges Montandon soutient encore ce topos, admis dans la communauté scientifique française: "les crânes nègres africains des deux sexes ont quelque chose de gracile qui peut faire croire, pour les deux sexes, à la féminité " (Montandon 1933: 73). Les similitudes physiques et intellectuelles entre les sexes sont en outre considérées comme répandues chez les populations indigènes: "C'est d'ailleurs un fait bien connu que, chez beaucoup de peuples sauvages ou seulement barbares, la constitution de la femme diffère bien moins que chez nous de celle de l'homme [...] les différences sexuelles sont d'autant moindres que l'état social est plus voisin de la barbarie ", lit-on dans le Dictionnaire encyclopédique des sciences médicales en 1877 (Dally 1877 : 437). En 1898, pour Gustave Le Bon, versé dans l'anthropologie et la sociologie à la fin du XIX ${ }^{e}$ siècle, les hommes et les femmes des peuples sauvages se ressembleraient d'un point de vue anatomique et intellectuel. Ce serait, en outre, une caractéristique qui distinguerait la sauvagerie de la civilisation. Cette dernière aurait pour effet d'accroître le dimorphisme sexuel ainsi que l'écart intellectuel entre les hommes et les femmes.

6 La hiérarchie des rôles sociaux entre les sexes ne semble en outre pas établie en Afrique de la même manière qu'en Europe, entraînant une confusion entre les genres. En effet, la supposée oisiveté des hommes, mise en contraste avec le travail des femmes, décrit comme harassant, est critiquée par les savants français (Hovelacque 1889, Verrier 1892, Letourneau 1903). Selon eux, ces situations virilisent les femmes, physiquement et moralement, affaiblissent et efféminent les hommes et désorganisent ainsi le fonctionnement de la société. La féminisation de l'homme noir dans les écrits médicaux contribue donc à dénier à ce dernier sa capacité à gouverner et à imposer son autorité sur les membres de sa communauté. Par un jeu de transposition, la nation française s'apparente à l'homme dans la pensée savante du XIX siècle tandis que les sociétés 
africaines sont assimilées à la femme. Un rapport de pouvoir, favorable aux Français, s'établit donc dans les textes scientifiques puis dans le langage politique, sous la voix de Jules Ferry à partir des années 1880 notamment.

7 La sexualité constituerait également un domaine dans lequel l'homme et la femme noirs se distingueraient peu. Pour les médecins, les penchants sexuels irrépressibles des Africaine's s'expliqueraient par la conformation de leur cerveau. Cette activité sexuelle démesurée expliquerait le surdéveloppement de leurs attributs sexuels. Le stéréotype du surdimensionnement des organes génitaux dans la race noire se voit donc accrédité et diffusé par la caution scientifique. L'homme africain est en outre perçu, comme la femme, comme un être faible, soumis à son sexe envahissant et à sa sexualité. Il est par ailleurs menacé par le satyriasis, l'équivalent de la nymphomanie chez la femme. Dans la définition de ce terme, donnée dans le Dictionnaire encyclopédique des sciences médicales en 1879, une catégorie d'hommes est décrite comme étant particulièrement soumise à cette maladie : «le nègre obéit à ses sensations, et n'est occupé que de satisfaire sa faim, on le voit s'abandonner sans réserve aux plaisirs sexuels » (Bouchereau 1879: 67). À la même époque, le Grand Dictionnaire universel du $\mathrm{XIX}^{e}$ siècle de Pierre Larousse, destiné à un large public, relaie ce préjugé : «Les femmes, comme les hommes, de la race nègre sont portées à la lasciveté beaucoup plus que les femmes blanches. La nature semble avoir accordé aux fonctions physiques ce qu'elle a refusé aux fonctions intellectuelles de cette race " (Virey 1815: 513, Larousse 1872 : 203). La sexualité imprimerait sa marque sur le corps et l'esprit des Noire's. L'homme, maîtrisant ses pulsions et ses passions chez les peuples civilisés, serait subordonné à son corps et à ses excès dans les peuples primitifs. Dans les colonies, l'homme noir incarnerait le corps tandis que l'homme blanc représenterait le cerveau.

D'autres stigmates, imprimés sur l'anatomie des Africains, semblent témoigner pour les médecins d'une absence de virilité dans la race noire. Les nombreux discours sur la pilosité africaine et plus particulièrement sur l'aspect glabre de leurs corps véhiculent l'image d'une virilité amoindrie, défaillante voire inexistante chez les hommes noirs. Le système pileux constitue en effet l'un des principaux attributs de la virilité chez l'homme européen, avec le port de la barbe notamment, alors que la chevelure constitue l'essence de la féminité (Sohn 2009, Corbin, Courtine et Vigarello 2011). Du point de vue des normes esthétiques européennes, le brouillage des différences sexuelles semble marqué en Afrique. Les hommes y sont décrits comme imberbes et les femmes avec une chevelure rasée ou inexistante. Dans l'œuvre de l'écrivain A. Dulaure parue en 1786, les Lapons, les Japonais et les Indiens sont présentés comme des hommes imberbes et par conséquent efféminés, à l'instar des Africains privés par la nature de cet attribut. Les Européens, privilégiés par une complexion pourvoyeuse de pilosité, sont pour leur part considérés comme des êtres supérieurs: "Comme un visage sans barbe est l'indice de l'enfance et de la faiblesse, un menton barbu, celui de la virilité et de la prudence » (Dulaure 1786 : 99). L'utilisation de l'histoire permet à cet auteur de démontrer que la force s'est toujours rangée du côté des hommes barbus et l'impuissance du côté des hommes glabres. La pilosité faciale chez l'homme recouvre donc bien cette idée de force virile dans la pensée savante du XIX ${ }^{e}$ siècle tandis qu'elle incarne chez la femme la notion de désordre sexuel, moral et social :

Dans nos climats, les personnes les plus velues sont certainement, en général, les plus vigoureuses. On sait encore que les habitants indigènes [...] qui ont bien moins de poils que les Européens, passent pour être plus faibles que ces derniers...

(Villermé 1827 : 187) 
Si l'absence de pilosité traduit la faiblesse et l'effémination de l'homme dans le savoir médical, elle est également considérée comme un caractère infantile. Au-delà de son statut de marqueur sexuel et social, le système pileux d'un homme traduirait sa place sur l'échelle humaine. Pour le docteur Clavel, chez « les nègres de l'Afrique centrale », "l'homme est imberbe [...] dans ses mœurs, sa langue, ses goûts et son intelligence, tout est enfantin. Au contraire, la virilité éclate de toutes parts chez les peuples des contrées froides " (Clavel $1860: 57$ ). Le tempérament lymphatique dont seraient dotés les hommes noirs, un tempérament jugé propre aux femmes, serait l'une des causes de leur absence de pilosité. Contrairement au tempérament sanguin des hommes blancs, à l'origine de leur puissance, il expliquerait leur mollesse et leur paresse, ainsi que leur soumission au colonisateur de race blanche. La dénégation de la puissance masculine en Afrique permet en outre de renforcer l'image d'un colonisateur, puissant et viril, appartenant à une nation forte et conquérante, apportant à des peuples inconstants et inférieurs une protection paternelle (Guillaumin 1992, Connell 2005, Dorlin 2006). À cette race féminine, donnant l'impression d'être composée d'hommes dépourvus de virilité s'oppose donc la race masculine de la nation française, puissante et expansionniste, incarnée par des hommes forts, virils et conquérants. Ils concentreraient en eux la puissance sexuelle et le pouvoir, intrinsèquement liés dans les représentations. La colonisation se trouve donc légitimée par ces représentations sur le sexe, le genre et la virilité en Afrique. Elles sont diffusées par des agents de la colonisation, médecins de la marine ou des colonies, mais également par des médecins et anthropologues de cabinet en métropole, dotés du savoir et du pouvoir en tant que scientifiques (Léonard 1981). Ce discours n'est toutefois pas uniforme, homogène et linéaire tout au long de la période. Conjointement à ces représentations, se sont diffusés d'autres préjugés sur le corps de l'homme noir et son hyper-virilité, due à sa force physique jugée exceptionnelle et à sa prétendue résistance face aux maladies. Cette virilisation de l'homme noir est apparue dans les écrits scientifiques à un moment où la nécessité d'imposer un modèle "naturel » aux hommes blancs affaiblis par la civilisation s'est avéré nécessaire et que le recrutement d'Africains dans les troupes coloniales est devenu impératif. Cette perception de la force masculine noire se développe en effet dès la fin du siècle et emprunte quelques-uns de ses traits à la période de l'esclavage et de la traite négrière. Dans cette vision qui se superpose à la précédente pour s'y substituer peu à peu dans la première moitié $\mathrm{du} \mathrm{xx}^{\mathrm{e}}$ siècle, la virilité ne se range plus uniquement du côté de la civilisation, elle se place du côté de la nature.

\section{La re-virilisation de l'Africain}

9 Le développement du courant naturiste et des besoins de la colonisation au tournant des $\mathrm{XIX}^{\mathrm{e}}$ et $\mathrm{xx}^{\mathrm{e}}$ siècles contribue à diffuser, au sein de la littérature médicale, mais également dans les représentations populaires, l'image d'une virilité accomplie de l'homme noir. Son corps musclé, robuste, endurant et fort est alors loué face au corps affaibli de l'homme blanc dans les colonies. La « force noire » devient indispensable à la réussite du projet colonial. Il s'agit donc de légitimer l'exploitation d'hommes jugés supérieurs physiquement au colonisateur. La forte résistance des Africains aux climats chauds, leur acclimatation parfaite, comparée à celle, chancelante, des expatriés européens amoindris sous les climats africains, font d'eux des êtres « utiles » comme main-d'œuvre ou comme soldats (Berger $1870:$ 443-450). La célèbre «force noire » du 
général Mangin, vantée en 1910, s'appuie sur un stéréotype puissant, hérité de l'ère esclavagiste : celui de la résistance physique des Africains. Ce préjugé renforce toutefois une autre idée reçue, celle de la prééminence du corps sur l'esprit chez les Africains. Tandis que la puissance corporelle est attribuée aux hommes noirs, le pouvoir de décision est confié aux hommes blancs. Cette représentation est par ailleurs véhiculée par des praticiens de terrain comme le docteur Bérenger-Féraud. La prétendue invulnérabilité des Noirs en milieu africain représente pour ce médecin de la Marine un atout dont la colonisation doit profiter afin d'assurer l'exploitation de la colonie :

les Ouolofs constituent quoi qu'il en soit, une race qui peut nous servir beaucoup dans notre œuvre de colonisation de la Sénégambie. Il nous faut nous en servir avec habileté pour mettre le pays en rapport, et en créant des besoins à ce peuple d'une part, tandis que nous les dirigerons dans le sens propice à notre intérêt de civilisation, il constituera une classe inférieure de travailleurs et de producteurs qui aura certainement son utilité, parce qu'il peut vivre dans un milieu qui nous est éminemment nuisible et par conséquent qu'il peut nous suppléer dans quelques occupations. (Bérenger-Féraud $1879: 62$ )

Aristide Le Dantec, médecin militaire français, créateur et premier directeur de l'école de médecine de l'AOF à Dakar, diffuse cette idée au début du Xxe siècle dans ses cours de pathologie exotique: "L'Européen dans les pays chauds trouve dans l'indigène un auxiliaire indispensable pour la main-d'œuvre que lui-même n'a pas toujours la force d'accomplir en raison de sa santé précaire " (Le Dantec 1902: 15). L'indigène apparaît donc comme un être fort et résistant dans ces discours.

Ces craintes autour de l'affaiblissement de l'homme blanc ne se circonscrivent toutefois pas au milieu colonial. De nombreux discours, émanant de la sphère médicale mais aussi politique en France, attestent d'une peur de la dégénérescence de la race blanche. La défaite de 1870-1871 face à la Prusse a marqué les esprits. Elle est interprétée comme le signe d'un avilissement des membres, masculins, de la société française. Ces derniers sont perçus comme affaiblis et corrompus par les vices de la civilisation, de la ville et de l'industrialisation. Un retour à la nature est donc prôné. Dans ce contexte, l'indigène est présenté comme un homme robuste et préservé. La virilité de l'homme noir s'apparente à une force naturelle perdue par les civilisés. Si le modèle rousseauiste du bon sauvage imprègne la pensée européenne au tournant des $\mathrm{XVIII}^{\mathrm{e}}$ et $\mathrm{XIX}^{\mathrm{e}}$ siècles, les hommes noirs étant perçus comme des individus musclés, purs et sains, ces représentations trouvent un nouvel écho à la Belle Époque avec le développement du naturisme. Le rapprochement de l'Homme avec la nature est vu, dans cette doctrine néo-hippocratique, comme un moyen efficace de lutter contre les dangers de l'urbanisation et de l'industrialisation (Baubérot 2004, Villaret 2005). Le docteur Rouhet $(1902,1913)$ comme le docteur Carton militent dès les années 1900 en faveur de la médecine naturiste. À cette époque, le corps musclé de l'homme devient un signe de santé et de virilité. La saillie des muscles doit incarner la puissance corporelle. L'humiliation de la défaite des Français face aux Prussiens, attribuée en partie à la faiblesse physique et morale des hommes, contribue en cette fin de siècle au développement des pratiques sportives, comme la gymnastique, dans le but de renforcer les corps et de les rendre aptes au combat (Vigarello [1979] 2001). Dans ce contexte, les écrits médicaux sur les races humaines valorisent les anatomies musclées et puissantes des Africains. Le docteur Mondière, médecin de première classe de la marine, vante leur robustesse physique, notamment sur la côte occidentale : « La force musculaire, entretenue par un travail assidu, est assez considérable, surtout comme résistance à la fatigue " (Mondière 1880: 628). Plusieurs médecins de brousse 
témoignent, dans leurs rapports, de la musculature hors du commun des hommes qu'ils côtoient (Gaillard et Poutrin 1914: 23). Au moment même où les chantres de la civilisation vantent ses vertus et la nécessité d'aller diffuser les Lumières aux peuples indigènes, d'autres penseurs critiquent ses vices et font l'éloge des qualités des Africains et notamment de leur force virile. Ce n'est en outre plus seulement l'inné ou la race qui est en cause mais leur mode de vie. En effet, les discours sur les facteurs acquis se développent, sous l'influence de l'anthropologie culturaliste américaine et de la naissance de l'ethnographie en France, et contrebalancent les théories innéistes au tournant des XVIII ${ }^{e}$ et $\mathrm{XIX}^{\mathrm{e}}$ siècles. Les mœurs des indigènes deviennent parfois des exemples à suivre pour redresser le corps des Européens. L'officier de marine Georges Hébert (1875-1957) est l'un de ceux qui prônent cet exemple. Il choisit en effet de s'inspirer des pratiques physiques des indigènes pour établir un programme d'éducation sportive dite naturelle à destination des Français en 1907. Il est expérimenté à l'École des fusiliers marins de Lorient et deviendra, par la suite, la méthode nationale sous le gouvernement de Vichy en 1940. Être fort, robuste et puissant grâce à une vie "naturelle ", à la manière des Africains, doit donc permettre aux Français d'assurer la défense et la protection de leur nation. Les hommes blancs doivent se régénérer dans un retour à la nature afin de renforcer leur corps.

11 Cette re-virilisation est également perceptible à une période où «la force noire " présente un intérêt essentiel pour la préservation de la nation française. Le corps des tirailleurs est créé en 1857 par le général Faidherbe, gouverneur du Sénégal, pour combattre dans les territoires colonisés dans un premier temps. Le "tirailleur sénégalais » est celui qui symbolise cette force africaine louée par les médecins et les administrateurs coloniaux (Fargettas 2012, Deroo et Champeaux 2006, Michel 2003). Dans le dernier tiers du siècle, de nombreux hommes de terrain préconisent la mise sur pied d'une armée coloniale capable de servir la France sur le territoire métropolitain. La mise en exergue de la robustesse africaine constitue d'ailleurs un argument central dans leurs discours (Reynaud 1892 : 51). C'est le général Mangin qui donne l'impulsion à l'utilisation de cette « manne » humaine au sein des rangs de l'armée française à partir de 1910 (Mangin 1910). Son argumentaire reprend celui des théoriciens de la dégénérescence tels qu'Adolphe Pinard ou Jacques Bertillon. «L'abâtardissement de la race » française, ne peut " plus remplir les anciennes conditions exigées pour le service militaire » pour le docteur Morel en 1857 (Morel 1857 : 9). Pour Mangin, il faut donc puiser la force là où elle se trouve, c'est-à-dire dans le corps des hommes noirs. Plus de 180000 tirailleurs d'Afrique Occidentale Française (AOF) et d'Afrique Équatoriale Française (AEF) sont recrutés lors de la Grande Guerre même si tous ne combattent pas sur le sol français. Environ 70000 de ces hommes sont morts lors de cette guerre. La loi sur le recrutement des troupes indigènes est promulguée en 1919. Elle rend le service militaire, d'une durée de trois ans, obligatoire, et organise les conditions de la mobilisation en Afrique. Toutefois, dans le raisonnement des médecins militaires, des commandants ou des généraux qui sont favorables à l'emploi de cette force indigène, le commandement doit demeurer entre les mains des hommes blancs, doués d'intelligence, tandis que les hommes noirs doivent représenter un vivier de soldats utiles pour leur résistance physique. Cet éloge de la puissance et de la masculinité des Africains réduit encore ces hommes à leur corps, un corps fort, vigoureux et vertueux. 

écrits médicaux qui ont pourtant un but commun : servir la France. Selon le contexte politique, la virilité de l'homme noir semble défaillir ou au contraire se renforcer. Durant la phase de conquête et d'expansion coloniale, la virilité du colonisateur se voit mise en exergue pour justifier sa prise de pouvoir, face à des hommes efféminés et faibles. La nation à laquelle il appartient apparaît comme dominante, puissante et protectrice. Toutefois, la virilité africaine recouvre ses droits lorsqu'il s'agit de présenter un exemple de vie naturelle aux hommes blancs affaiblis en métropole et surtout lorsqu'il faut leur trouver des suppléants sur le terrain colonial. Elle est également revalorisée lorsqu'il s'agit de vanter la force noire capable de vanter et de servir la nation française. Si la résistance physique est soulignée pour les hommes noirs, qui retrouvent ainsi une part de virilité souvent déniée au XIX ${ }^{e}$ siècle, la femme africaine recouvre également une part de féminité dans les écrits sur la maternité. L'accomplissement jugé exemplaire de leur mission maternelle, lié à une vie naturelle, contribue à les ériger en modèles pour les femmes blanches, accusées de se détourner de leur destinée au cours du XIX ${ }^{\mathrm{e}}$ siècle (Virey 1824, Mondière 1881, Sarrazin 1901, Letourneau 1903). La virilité et la féminité, constructions sociales et culturelles, recourent donc parfois à des modèles "naturels" pour tenter de revigorer les membres, masculins et féminins, de la nation française.

\section{BIBLIOGRAPHIE}

Baubérot, Arnaud, 2004, Histoire du naturisme. Le mythe du retour à la nature, Rennes, PUR.

Connell, Raewyn, 2005, Masculinities, Los Angeles, University of California Press.

Corbin, Alain, Courtine, Jean-Jacques et Vigarello, Georges, 2011, Histoire de la virilité, t. II, Paris, Seuil.

Deroo, Éric et Champeaux, Antoine, 2006, La Force noire. Gloire et infortunes d'une légende coloniale, Paris, Tallandier.

Dorlin, Elsa, 2006, La Matrice de la race. Généalogie sexuelle et coloniale de la nation française, Paris, La Découverte.

Fargettas, Julien, 2012, Les Tirailleurs sénégalais. Les soldats noirs entre légendes et réalités (1939-1945), Paris, Taillandier.

Guillaumin, Colette, 1992, Sexe, race et pratique du pouvoir, Paris, Côté-femmes.

Léonard, Jacques, 1981, La Médecine entre les savoirs et les pouvoirs. Histoire intellectuelle et politique de la médecine française au XIX siècle, Paris, Aubier Montaigne.

Michel, Marc, 2003, Les Africains et la Grande guerre. L'appel à l'Afrique (1914-1918), Paris, Karthala.

Sohn, Anne-Marie, 2009, Sois un homme! La construction de la masculinité au XIX ${ }^{e}$ siècle, Paris, L'Univers historique.

Itinéraires, 2019-2 et 3 | 2019 
Revenin, Régis (dir.), 2007, Hommes et masculinités, de 1789 à nos jours : contributions à l'histoire du genre et de la sexualité en France, Paris, Autrement.

Sinha, Mrinalini, 1995, Colonial Masculinity. The "Manly Englishman" and the "Effeminate Bengali" in the late Nineteenth Century, Manchester, Manchester University Press.

Taraud, Christelle, 2011, «La virilité en situation coloniale, de la fin du XVIII ${ }^{\mathrm{e}}$ siècle à la Grande Guerre », dans A. Corbin, J.-J. Courtine et G. Vigarello, Histoire de la virilité, t. II, Paris, Seuil, p. 331-347.

Villaret, Sylvain, 2005, Naturisme et éducation corporelle, Paris, L'Harmattan.

Vigarello, Georges, [1979] 2001, Le corps redressé, Paris, Armand Colin.

\section{Corpus}

Bérenger-Féraud, Laurent Jean-Baptiste, 1879, Les Peuplades de la Sénégambie, Paris, Ernest Leroux. Berger, Charles-Victor, 1870, « Considérations hygiéniques sur les tirailleurs sénégalais », Archives de médecine navale, $\mathrm{n}^{\circ}$ 14, Paris, J. B. Baillière.

Bouchereau, Gustave, 1879, « Satyriasis », dans Dictionnaire encyclopédique des sciences médicales, t. VII, Paris, A. Dechambre.

Broc, Pierre-Paul, 1836, Essai sur les races humaines considérées sous les rapports anatomique et philosophique, Paris, Librairie des Sciences médicales, De Just Rouvier et E. Le Bouvier.

Broca, Paul, 1861, Sur le volume et la forme du cerveau suivant les individus et les races, Paris, Typographie Hennuyer.

Cabanis, Pierre-Jean-Georges, 1802, Rapports du physique et du moral de l'homme, Tome premier, Paris, Chez Crapart.

Clavel, Adolphe, 1860, Les Races humaines et leur part dans la civilisation, Paris, Poulet-Malassis et De Broise éditeurs.

Dally, Eugène, 1877, « Femmes », dans A. Dechambre (dir.), Dictionnaire encyclopédique des sciences médicales, Tome premier, quatrième série, Paris, G. Masson.

Dulaure, Jacques-Antoine, 1786, Pogonologie ou Histoire philosophique de la barbe, Constantinople, Paris, Le Jay.

Gaillard, Raoul et Poutrin, Léon, 1914, «Étude anthropologique des populations des régions du Tchad et du Kanem », dans Documents scientifiques de la mission Tilho (1906-1909), Tome troisième, Paris.

Hovelacque, Abel, 1889, Les Nègres de l'Afrique sus-équatoriale (Sénégambie, Guinée, Soudan, Haut-Nil), Paris, Legrosnier et Babé.

Larousse, Pierre, 1872, « Femme », Grand dictionnaire universel du XIX siècle, t. VIII, Paris, Administration du Grand Dictionnaire Universel.

Le Bon, Gustave, 1898, Lois psychologiques de l'évolution des peuples, Paris, Félix Alcan.

Le Dantec, Aristide, 1902, Les Colonies, introduction à l'étude de la pathologie exotique, Paris, Imprimerie F. Levé.

Lefrou, Gustave, 1943, Le Noir d'Afrique. Antropo-biologie et raciologie, Paris, Payot. 
Letourneau, Charles, 1869, « Caffres », dans A. Dechambre (dir.), Dictionnaire encyclopédique des sciences médicales, t. XI, Paris, Masson.

Letourneau, Charles, 1903, La Condition de la femme dans les diverses races et civilisations, Paris, V. Giard et E. Brière libraires-éditeurs.

Mangin, Charles, 1910, La Force noire, Paris, Hachette.

Mondière, Alfred Théophile, 1881, « Les nègres chez eux ou études ethnographiques sur les populations de la Côte d'Or (Côte Occidentale d'Afrique) », Revue d'Anthropologie, Série 2, t. 3.

Montandon, George, 1933, La Race, les races. Mise au point d'ethnologie somatique, Paris, Payot.

Morel, Benedict-Augustin, 1857, Traité des dégénérescences physiques, intellectuelles et morales de l'espèce humaine, Paris, J.B. Baillière.

Pittard, Eugène, 1927, « Contribution à l'étude craniologique des Griquas », L'Anthropologie, t. 37, Paris, Masson et Cie.

Reynaud, Gustave, 1892, "L'armée coloniale au point de vue de l'hygiène pratique ", Archives de médecine navale et coloniale, $\mathrm{n}^{\circ} 58$, Paris, Octave Doin.

Rouhet, Georges, 1902, De l'entraînement complet et expérimental de l'homme, Paris, Libraires associés.

Rouhet, Georges, 1913, Revenons à la nature et régénérons-nous, Paris, Berger-Levrault.

Sarrazin, Henri, 1901, Races humaines du Soudan français, Chambéry, Imprimerie générale de Savoie.

Topinard, Paul, 1879, L'Anthropologie, Paris, C. Reinwald et Cie, $3^{\mathrm{e}}$ édition.

Verrier, Eugène, 1892, "Étude sur le sort de la femme en Afrique », Bulletins et Mémoires de la Société Africaine de France, Paris, A. Challamel.

Villermé, Louis-René, 1827, « Poils », dans Encyclopédie méthodique, médecine, par une société de médecins, Tome douzième, Paris, Chez Mme veuve Agasse.

Virey, Julien-Joseph, 1815, « Femme », dans Dictionnaire des sciences médicales, t. XIV, Paris, Panckoucke éditeur.

Virey, Julien-Joseph, 1817, « Homme », dans Dictionnaire des sciences médicales, vol. XXI, Paris, Panckoucke.

Virey, Julien-Joseph, 1824, Histoire naturelle du genre humain, t. II, Paris, Crochard libraire-éditeur.

\section{RÉSUMÉS}

Le corps de l'homme noir est soumis à deux types de représentations dans la littérature médicale française $d u \mathrm{XIX}^{\mathrm{e}}$ siècle jusqu'au milieu $d u \mathrm{xx}^{\mathrm{e}}$ siècle. Ces descriptions s'établissent souvent en miroir de celles de l'homme blanc et contribuent à renforcer la virilité de ce dernier. En effet, l'homme africain est décrit par les médecins comme un être efféminé, physiquement et intellectuellement, proche de la femme et de l'enfant, inférieur et dénué d'autorité. Cette infériorité semble en outre légitimer la colonisation des terres africaines par les hommes de la nation française, présentés quant à eux comme virils et puissants, et censés apporter une protection à ces peuples. Parallèlement à cette représentation coexiste un autre topos qui se renforce au tournant des $\mathrm{XIX}^{\mathrm{e}}$ et $\mathrm{XX}^{\mathrm{e}}$ siècles du fait du développement du courant naturiste et des besoins de la colonisation, celui d'une hyper-virilité de l'homme noir. Son corps musclé, robuste, 
endurant et fort est alors loué face au corps affaibli de l'homme blanc dans les colonies. Ce dernier y est en effet menacé par le climat et les pathologies tropicales tandis qu'en métropole l'on s'inquiète de la dégénérescence de la race après la défaite de 1870-1871. La définition de la virilité africaine varie donc au cours du $\mathrm{XIX}^{\mathrm{e}}$ siècle et des débuts $\mathrm{du} \mathrm{XX}^{\mathrm{e}}$ siècle selon le contexte politique et les normes sexuelles et sociales en vigueur en France.

The black man's body is subject to two types of representations in the French medical literature from the nineteenth century to the middle of the twentieth century. Descriptions of black men often contributed to emphasizing the virility of white men. Indeed, African men were described as physically and intellectually effeminate, similar to women and children, inferior and lacking authority. This inferiority was used to legitimize the colonization of Africa by men of the French nation, who were presented as manly and powerful, and thus able to protect the African population. Parallel to this representation, another stereotype emerged at the turn of the nineteenth and twentieth century with the development of the naturist doctrine and colonization, this time foregrounding the hyper-virility of black men. The representation of his robust, resistant, strong and muscular body, was contrasted to the weakened body of white men, at a time when, in the colonies, white men suffered from the climate and tropical diseases and, in France, the defeat of 1870 led to rising fear about the "decline of the race". So, the definition of black man's masculinity evolves during the nineteenth century and the beginnings of the twentieth century according to the political context and the sexual and social norms prevailing in France.

\section{INDEX}

Mots-clés : corps, corps noirs, virilité, médecine, colonisation, nation française, littérature médicale

Keywords : bodies, Black bodies, virility, medicine, colonisation, French nation, medical literature

\section{AUTEUR}

\section{DELPHINE PEIRETTI-COURTIS}

Aix-Marseille Université, TELEMMe 